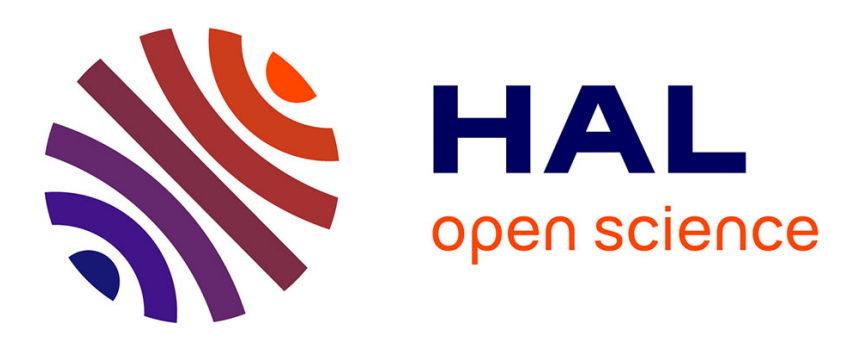

\title{
A latent profile analysis revealed different categories of patients in anorexia nervosa: proposal of guidelines in adapted physical activity
}

Laurence Kern, Nathalie Godart, Laura Tailhardat, Amandine Peguet, Marie Grall-Bronnec, Lilian Fautrelle

\section{To cite this version:}

Laurence Kern, Nathalie Godart, Laura Tailhardat, Amandine Peguet, Marie Grall-Bronnec, et al.. A latent profile analysis revealed different categories of patients in anorexia nervosa: proposal of guidelines in adapted physical activity. Movement \& Sport Sciences - Science \& Motricité, 2019, 103, pp.55-66. 10.1051/sm/2018022 . hal-02311172

\section{HAL Id: hal-02311172 \\ https://hal.parisnanterre.fr/hal-02311172}

Submitted on 10 Oct 2019

HAL is a multi-disciplinary open access archive for the deposit and dissemination of scientific research documents, whether they are published or not. The documents may come from teaching and research institutions in France or abroad, or from public or private research centers.
L'archive ouverte pluridisciplinaire HAL, est destinée au dépôt et à la diffusion de documents scientifiques de niveau recherche, publiés ou non, émanant des établissements d'enseignement et de recherche français ou étrangers, des laboratoires publics ou privés. 


\title{
A latent profile analysis revealed different categories of patients in anorexia nervosa: proposal of guidelines in adapted physical activity
}

\author{
Laurence Kern ${ }^{1,2}$, Nathalie Godart ${ }^{3,4}$, Laura Tailhardat ${ }^{2}$, Amandine Peguet ${ }^{2}$, Marie Grall-Bronnec ${ }^{5,6}$, \\ and Lilian Fautrelle ${ }^{7,8, *}$ \\ ${ }^{1}$ Laboratoire EA 4430 CLIPSYD Clinique Psychanalyse Développement, Université Paris Nanterre, Nanterre, France \\ ${ }^{2}$ Laboratoire EA 2931, CeSRM, Université Paris Nanterre, Nanterre, France \\ ${ }^{3}$ CESP, Fac. de médecine-Univ. Paris-Sud, Fac. de médecine-UVSQ, INSERM, Université Paris-Saclay, 94805 Villejuif, France \\ ${ }^{4}$ Service de psychiatrie, Institut Mutualiste Montsouris, Université Paris Descartes, Faculté de Médecine, Paris, France \\ ${ }^{5}$ CHU Nantes, service d'Addictologie, Nantes, France \\ ${ }^{6}$ Université de Nantes, Université de Tours, UMR1246, Nantes, France \\ ${ }^{7}$ Institut National Universitaire Champollion, STAPS, Équipe interdisciplinaire en activités physiques, Campus de Rodez, \\ 12000 Rodez, France \\ ${ }^{8}$ Laboratoire ToNIC, Toulouse NeuroImaging Center, Université de Toulouse, Inserm, UPS, Toulouse, France
}

Received 13 January 2018, Accepted 26 October 2018

\begin{abstract}
Anorexia nervosa (AN) is frequently associated with anxiety, depression, and problematic exercise (PE). These uncontrolled physical activities inducing resistance to the therapeutic protocols. Some recent studies demonstrated that adding controlled and adapted physical activities (APA) in the therapeutic protocol was more positive for AN patients than the usual physical activity suppression. The aim of the present study was to highlight different profiles of AN patients in order to better individually adapt the physical activity programs, and thus the therapeutic impact of the future APA programs. Problematic exercises, cardio-respiratory fitness, perceived fitness and mood were measured in fifty-two AN patients [all females, $17.15( \pm 2.22)$ years old, BMI $=16.49$ $( \pm 1.54)]$ from three different French therapeutic centers. Then, a latent profile analysis allowed to exhibit 3 classes corresponding to 3 different profiles of AN patients. A first patient profile was characterized by proven PE with lower anxiety and depression. A second profile exhibited a moderate PE coupled with a high level of anxiety and depression. The last profile revealed patients without any PE, and medium scores in the other variables. These results lead to propose different angles and objectives to design and improve future APA programs according to these patient profiles.
\end{abstract}

Key words: anorexia nervosa, problematic exercise, mood, latent profile analysis, adapted physical activity

Résumé- Une analyse en classes latentes révèle différentes catégories de patients dans l'anorexie mentale: proposition de lignes directrices en matière d'activité physique adaptée. Les patients souffrant d'anorexie mentale (AM) présentent souvent des symptômes d'anxiété, de dépression, et une pratique problématique d'activité physique (PPAP). Cette PPAP induit une résistance aux protocoles thérapeutiques. En revanche, des études récentes ont démontré que l'utilisation d'activités physiques adaptées (APA) était meilleure pour ces patientes qu'une privation totale d'exercice physique. L'objectif de cette étude est de mettre en lumière différents profils de patientes AM afin de permettre une meilleure individualisation des contenus des programmes d'APA. La PPAP, la condition physique perçue, l'adaptation cardiaque à l'effort, les niveaux d'anxiété et de dépression ont été mesurés chez cinquante-deux patientes anorexiques $[17,15( \pm 2,22)$ ans, $\mathrm{IMC}=16,49( \pm 1,54)]$. Ensuite, une analyse en classes latentes des scores a permis de mettre en lumière 3 profils cliniques significativement différents. Le premier profil est caractérisé par une PPAP totale associée à de faibles niveaux d'anxiété et de dépression. Le second est caractérisé par une PPAP symptomatique, mais un très haut niveau d'anxiété et de dépression. Enfin, le troisième profil présente des patientes sans PPAP et des scores moyens dans les autres variables. Ces résultats nous conduisent à proposer différents angles et objectifs pour réaliser des programmes d'APA en fonction du type de profil des patientes prises en charge.

Mots clés : anorexie mentale, pratique problématique d'activité physique, analyse en classes latentes, activités physiques adaptées

\footnotetext{
*Corresponding author: lilian.fautrelle@inserm.fr
} 


\section{Introduction}

Anorexia nervosa (AN) is a very common chronic disease (Patton, Coffey, \& Sawyer, 2003). Occurring during adolescence, AN can also extend into adulthood. This disorder induces mortality risk multiplied by almost 20 including a high suicide rate (Steinhausen, 2009), making AN the psychiatric disorder with the highest mortality rate (Franko et al., 2013), and with a standardized mortality ratio (SRM) of 5.9 (Arcelus, Mitchell, Wales, \& Nielsen, 2011). The main abnormal behaviour from affected patients is characterized by partial or total restrictive eating during months or years in order to minimize their body-weight, despite an already normal or low body-weight (American Psychiatric Association, 2013). Such a behaviour is related to disruptions with body shape perception and self-esteem (Woodward, McIlwain, \& Mond, 2017). The sustainable food deprivations or vomiting and purgative abuse and their inherent symptoms - malnutrition, underweight, amenorrhoea - could induce multiple somatic complications (van Elburg \& Danner, 2015). It is possible to find among them: neuronal damages (Ehrlich et al., 2008), cardiac disorders (Kuwabara, Niwa, Yamada, \& Ohta, 2017), impaired cardiovascular response (Lands, Pavilanis, Charge, \& Coates, 1992), reduced muscle force (Mueller et al., 2015), osteoporosis or fragile bones (Drabkin, Rothman, Wassenaar, Mascolo, \& Mehler, 2017) or renal injury (Stheneur et al., 2017). Moreover, AN is also very frequently associated with psychiatric symptoms and disorders as depression, anxiety (Junne et al., 2016; Leblé, Radon, Rabot, \& Godart, 2017), obsessive compulsive disorder (Young, Rhodes, Touyz, \& Hay, 2013), and problematic physical activity exercising (PPAE) (Rizk et al., 2015).

AN patients and more generally patients affected by eating disorders are known to have the highest frequency of physical training and sport practicing compared to all other psychiatric disorders (Mangerud, Bjerkeset, Lydersen, \& Indredavik, 2014). This PPAE also called hyperactivity in the literature, is a psychiatric symptom commonly associated with 31 up to $80 \%$ of AN patients (Hebebrand et al., 2003). This wide range could be due to two main factors. First, there are numerous definitions provided in the literature for eating disorder patients. Second, no international consensus emerged from studies in the field of AN about excessive sport practice, excessive exercise or also hyperactivity. In the present paper, the term problematic exercise (PE) will be used according to the definition given by Rizk (2015). Indeed, these authors included both quantitative - duration, frequency, intensity - and qualitative criteria - compulsive practice, addiction to physical training - to determine the PE practice, which is not the case for the other definitions. In this way, physical activity practice becomes PE when:

- the training volume (frequency*intensity*duration) is important considering the malnutrition state, i.e. more than $6 \mathrm{~h}$ per week according to Davis et al. (1997);

- the main aim of the patients is to decrease negative affects related to diet and/or;

- physical activities become a compulsive practice.
Cognitive mechanisms leading to PE may vary. Some parts can result from conscious processes: in relation to disruptions of self-body-shape perception, AN patients could permanently try to reduce their body-weight and/or increase their muscle tone. Other parts may result from unconscious processes: PE becomes uncontrolled and compulsive practice with varying intensities across patients. Physical activity in AN patients is a composite phenomenon as demonstrated by Rizk (2015). This composite phenomenon explains that qualitative process of physical activity in AN patients remains complex. Indeed this physical activity may be:

- conscious, i.e. voluntary practiced in order to lose weight;

- unconscious, i.e. physical activity infiltrate everyday life without being perceived by the patients, or;

- a mixed solutions of both of them above.

Generally, physical activity is conscious at the beginning of the psychopathology, then become unconscious at least partly when malnutrition increases. Then, it becomes automatic and problematic practice without conscious decision from the patients. Both these conscious and unconscious processes evolve over time. The more the disease becomes chronic, the more physical activities become unconscious and compulsive (Rizk, Kern, Godart, \& Melchior, 2014).

If physical activity practices are recommended in healthy population to contribute to preserve health, nonadapted physical activities in $\mathrm{AN}$ patients generate additional systemic stress in addition to malnutrition. In a physiological point a view, $\mathrm{PE}$ in $\mathrm{AN}$ patients could induce multisystem troubles as cardiopulmonary (Urhausen, Gabriel, Weiler, \& Kindermann, 1998; Apor, Petrekanich, \& Számadó, 2009) or musculoskeletal problems (Boutin et al., 2015; El Ghoch, Bazzani, \& Dalle Grave, 2014). In a point of view of movement and sport sciences, most of AN patients use wrong and unsafe training situations and postures increasing risks of injury (Gottschall, Mills, \& Hastings, 2013; Lisman, O'Connor, Deuster, \& Knapik, 2013). For instance, to get tonic abdominis muscles and a flat stomach, some AN patients excessively practice abdominal crunches and leg-raises. However, these movements mainly involve the psoas major and iliacus but not the rectus abdominis (McCreary \& Provance, 1993). A highly risk of back injuries appear for biomechanical reasons (Nolte, Krüger, Els, \& Nolte, 2013) and such a chronical abdominal pressures contribute to pelvic organ prolapse (and thus round belly) or other pelvic floor dysfunctions as urinary incontinence (Almeida et al., 2016; Sung \& Hampton, 2009; Vitton et al., 2011). More generally in $\mathrm{AN}$, uncontrolled physical activity and PE increase caloric expenditure without any adaptation of caloric intake inducing resistance to the therapeutic protocols (Dalle Grave, Calugi, \& Marchesini, 2008). That is why the common practice dealing with $\mathrm{PE}$ for AN during hospital treatments used to be the physical activity suppression.

Nowadays, the balance sheet of this full restriction approach is not positive either. It is mostly very badly 
experienced by AN patients whose anxiety is consequently increasing (Junne et al., 2016). Moreover, Beumont, Arthur, Russell, \& Touyz (1994) reported increasing tensions and conflicts between staff and patients who were prohibited from physical activities. From a dichotomist point of view, there is some proofs in the literature that supervised and adapted physical activities could be safely integrated in the therapeutic plan to convey multiple benefits in AN patients (Cook et al., 2016; Ng, Ng, \& Wong, 2013). Indeed, studies during the last decades clearly demonstrated that adding adapted physical activities in the therapeutic protocol could facilitate weight gain (Calogero \& Pedrotty, 2004; Chantler, Szabo, \& Green, 2006), restore strength and muscle functions (Fernandez-del-Valle et al., 2014; Kolnes, 2017; Vancampfort et al., 2014), improve the body shape specially in the abdominal region (El Ghoch, Calugi, Pellegrini, Chignola, \& Dalle Grave, 2016; El Ghoch et al., 2013), reverse cardiac abnormalities (Krantz, Gaudiani, Johnson, \& Mehler, 2011), and finally, improve quality of life (Hausenblas, Cook, \& Chittester, 2008). Adding physical activities may become a therapeutic tool in AN if and only if objectives and contents of the program are adapted to the patients. In Vancampfort et al. (2014), physical activities programs with aerobic and resistance training or with aerobic, yoga and basic body awareness therapy presented significant benefit effects for patients. More precisely, the program with aerobic and resistance training induced an increasing of muscle strength, body mass index and body fat percentage. Moreover, the one with aerobic, yoga and basic body awareness therapy induced lowered scores of eating pathology and depressive symptoms. As previously shown, $\mathrm{PE}$ is linked with $\mathrm{AN}$, characterized by too high training volume or/and uncontrolled and compulsive practices depending on the type of AN patients (Rizk, 2015). In such cases, well-being and practice pleasure are left out. We usually developed adapted physical activities programs based on aerobic exercises and muscles building according to Vancampfort et al. (2014). These physical exercises were group practiced and called out cognitive dissonances according to Gouju (1993), in order to restore meaning and good physical training practice. In this way, a practical set of guidelines for the clinical management and therapeutic use of exercise in eating disorder patients as proposed by Cook et al. (2016) is very useful. These authors suggested in their third recommendation to "screen for exercise-related psychopathology", and more particularly to identify "individuals that endorse pathological attitudes and behaviors towards exercise". In other words, individualize the psychopathological diagnostic and individualize in consequence the content of the physical activity programs to each AN patient profile. That is why the main aim of the present study was to diagnostic different profiles of AN patients based on both attitude and behavior towards physical activity but also heart fitness and mood. These crucial parameters will allow to more individually adapt the physical activity programs, while always respecting the main principles highlighted by Cook et al. (2016) and Vancampfort et al. (2014).

\section{Method}

\subsection{Participants}

In this study, only females aged from 14 to 24 years old with an average body mass index (BMI) of $16.49( \pm 1.54)$ composed the sample. They come from three different French therapeutic centers [Institut Mutualiste Montsouris (Paris): 34 patients; Poissy pediatric hospital: 9 patients; Centre Hospitalier Universitaire de Nantes: 9 patients] volunteered for the experiment. All participants included had a perfect knowledge of spoken and written French. Informed consent was obtained from all participants and from all parents of minor participants according to the guidelines of the clinical ethic committee of the Institut Mutualiste Montsouris. The CEPAR (i.e. Committee for the Evaluation of Protocols and Research Assistance) number is 2016-002. It is the local ethical committee of the Institut Mutualiste Montsouris hospital which was established as a proof of ethics approval by an independent committee (Institutional Review Board) for this research, in accordance with French law.

\subsection{Data acquisition}

Tests and surveys were used to collect data. Participants were first asked to provide gender information, age, height, weight, and sociodemographic data. Eating disorders were evaluated using the self-questionnaire Eating Disorder Examination-Questionnaire (EDE-Q) (Fairburn \& Beglin, 1994). This questionnaire coming from the gold standard (EDE) includes twenty-two items categorized in four under dimensions (restraint, eating concern, shape concern, and weight concern). In this questionnaire only the item $\mathrm{n}^{\circ} 18$ is considered. It allowed indeed to measure compulsion to physical practices. The PE was measured by the Exercise Dependence ScaleRevised well-known to evaluate addiction to physical activity. This scale was initially published by Hausenblas \& Downs (2002) then translated into French and validated one more time by Kern (2007). In this questionnaire, twenty-one items that saturate on seven dimensions (i.e. tolerance, withdrawal, intention effect, lack of control, time, conflict, continuance) allowed to compute a totalscore. The Godin Leisure-Time Exercise Questionnaire (GLTEQ) was used to reliably measure the quantity of physical exercise and the exercise behavior of each participant (Godin, 2011; Godin, Jobin, \& Bouillon, 1986) The GLTEQ is a self-report instrument that assesses the frequency of strenuous, moderate, and mild leisure-time exercise done for at least 20 min during a typical week. A total exercise index (weekly metabolic equivalents) is calculated by weighing the frequency of each intensity and summing for a total score. An additional question allowed to measure the frequency of physical activity (never, rarely, sometimes, and often). 
The International Fitness Scale (IFIS) (Ortega et al., 2011) was conducted to estimate the perceived adolescents' fitness. This survey includes five Likert-scale questions ( $1=$ very poor, $5=$ very good) about the perceived adolescents' physical fitness-cardiorespiratory fitness (CRF), muscular fitness (MF), speed-agility (SP-AG), flexibility and overall-fitness in comparison with their friends' physical fitness. In addition, the Physical SelfPerception Profile (Fox \& Corbin, 1989; Ninot, Delignières, $\&$ Fortes, 2000) consist on a 25 items (ISP-25) to assess dimensions of global self-esteem, physical self-worth, sport competence, physical condition, attractive body and physical strength. For further investigations, objective measures of the heart-rates were conducted. More precisely, a Ruffier's protocol exercises was performed and Ruffiers's as well as Dickson's index were computed (Joussellin, 2007) to measure cardio-respiratory fitness for each participant. These index allow to determine a global qualification of the cardiac system adaptability to physical effort, usually also called cardio-respiratory fitness (Faik, Vanderhulst, Rossem, \& Devroey, 2017; Joussellin, 2007). Following a 5 minutes laying down period, the participants' heart rate was measured a first time (T1). Then participants were asked to realize 30 air-squats regularly in 45 seconds, arms outstretched in front of them. Heart rate was measured a second time just after those 45 seconds (T2). At last, participants were instructed to lie down one more time during one minute. At the end of this one, heart rate was measured a third time (T3). The Ruffier's index (Ri) was computed as following:

$$
R i=\frac{\mathbf{T} 1+\mathbf{T} 2+\mathbf{T} 3-\mathbf{2 0 0}}{\mathbf{1 0}} .
$$

The Dickson's index $(D i)$ was computed as following:

$$
D i=\frac{(\mathbf{T} 2-\mathbf{7 0})+2 *(\mathbf{T} 3-\mathbf{T} \mathbf{1})}{\mathbf{1 0}} .
$$

The lower the index, the better the cardio-respiratory fitness. Finally, the quality of life was evaluated with the Duke health profile (Guillemin, Paul-Dauphin, Virion, Bouchet, \& Briançon, 1997; Parkerson, Broadhead, \& Tse, 1990). This survey includes seventeen questions about health perception. This tool allows to compute a score from 0 to 100 in ten dimensions which are: physical health, mental health, social health, overall health, perceived overall health, self-esteem (from $0=$ very poor to $100=$ very good) anxiety, depression, pain, and incapacity $($ from $0=$ very good to $100=$ very poor $)($ Tab. 1$)$.

\subsection{Statistical methods}

Descriptive statistics were conducted using means and standard deviations (SD) for continuous variables, and using counts and percentages for categorical variables.

Latent class analysis (LCA) was performed using MPlus 7 (Muthén \& Muthén, 1998) to identify distinct homogenous subgroups of patients within the dataset based on standard values of the 8 continuous indicators
(PE, compulsion, physical activity, fitness self-perception, physical self-perception, Ruffier Dickson test, quality of life (anxiety and depression)). Parsimony and fit criteria were used to decide upon the best fitting model. In order to determine the number of classes underlying the sample, we compared the fit of models with an increasing number of classes. Akaike's Information Criteria (AIC), the Bayesian Information Criteria (BIC) and Adjusted BIC (aBIC) were used as indicators for the optimal number of classes. Model with lower AIC, BIC and aBIC values suggest better-fitting models (Lawler, Heary, \& Nixon, 2017). The Lo-Mendell-Rubin test (LMR) and the Bootstrap Likelihood Ratio Test (BLRT) were used to determine the maximum number of classes to consider, compared the estimated model with a model with one less class (k-1). A non-significant LMR or BLRT $p$-value indicates that the inclusion of an additional class does not result in a significant improvement in fit. We also compared the entropy. This fit measures the accuracy with which models classify individuals into their most likely latent class. Higher values (1) indicating greater precision and 0 indicating no predictive power. Differences between information criteria values lead to an evaluation of the strength of evidence of the best model compared to other candidate models (Berlin, Williams, \& Parra, 2014). Once the class number was chosen, each participant was assigned to a class based on their most likely latent class membership. Then, one-way analyses of variance (ANOVA) were conducted and $\chi 2$ tests were used to compare the profiles (independent variables) with regard to different variables. Then, planned adjusted comparisons between pairs of profiles were conducted using Bonferroni post hoc tests for continuous variables scores or z-tests for proportions. All statistical tests were two-tailed and the Type I error rate was fixed at $5 \%$. The LPA was performed using Mplus version 7 (Muthén \& Muthén, 1998 ) and the ANOVAs and $\chi 2$ tests using IBM SPSS Statistics version 22.0 (IBM Corp. Released, 2011). Interestingly, in order to allow comparisons between all the tests together and for a sake of clarity to plot Figure 1, Z-scores were computed for each test and item as following:

$$
\text { Zscore }=\frac{\boldsymbol{x}-\boldsymbol{\mu}}{\boldsymbol{\sigma}},
$$

where $x$ represented the individual's score; $\mu$ the general mean; $\sigma$ the general standard deviation (Tab. 2).

\section{Results}

\subsection{The population of patients suffering from anorexia nervosa remains very heterogeneous}

Fifty-two AN patients [all females, $17.15( \pm 2.22)$ years old, body mass index (BMI): $16.49( \pm 1.54)]$ composed the sample. The first prominent result was the very high heterogeneity which concerned many variables (please see Tab. 1). More precisely, the highest heterogeneities were found for the declared sedentary lifestyle $(372.69 \pm 251.67)$ 
Table 1. Descriptive results and statistics. The first table presents the descriptive scores for all the most important measures. The surveys and their items are given in the two firsts left columns in all the table. In the upper part of the table, from age to physical strength items, mean scores ( \pm standard deviation) as well as the minimum and the maximum values in each item are reported in the third and fourth column respectively. In the lower part of the table, the results by item were reported in percentage of the patient sample. In addition, the real number of patients is given into brackets.

\begin{tabular}{|c|c|c|c|}
\hline Test/survey & Item & Mean (SD) & Min-max \\
\hline \multirow{11}{*}{ Perceived fitness } & Age & $17.15(2.22)$ & $14-24$ \\
\hline & Body mass index & $16.49(1.54)$ & $13.05-20.76$ \\
\hline & GLTEQ & $35.13(24.02)$ & 3-96 \\
\hline & Sedentarity & $372.69(251.67)$ & $0-1080$ \\
\hline & General & $3.2(0.94)$ & $1-5$ \\
\hline & Cardio respiratory & $3.45(1.12)$ & $1-5$ \\
\hline & Strenght & $2.84(0.96)$ & $1-4$ \\
\hline & Speed & $2.71(0.92)$ & $1-5$ \\
\hline & Agility & $3.1(1.06)$ & $1-5$ \\
\hline & Flexibility & $3(1.27)$ & $1-5$ \\
\hline & Dickson's index & $4(3.21)$ & $-1-12$ \\
\hline \multirow{7}{*}{ Duke health profile (quality of life) } & Physical health & $65.77(20.03)$ & $20-100$ \\
\hline & Mental health & $43.33(22.86)$ & $0-80$ \\
\hline & Perceived health & $39.22(39.13)$ & $0-100$ \\
\hline & Self-esteem & $52.2(19.3)$ & $10-90$ \\
\hline & Anxiety & $50(20.69)$ & $8.33-91.66$ \\
\hline & Depression & $46.8(22.9)$ & $10-100$ \\
\hline & Pain & $42.31(31.91)$ & $0-100$ \\
\hline \multirow{8}{*}{ Exercise dependence scale } & Withdrawal & $3.33(1.47)$ & $1-5.67$ \\
\hline & Continuance & $2.68(1.5)$ & $1-6$ \\
\hline & Tolerance & $3.4(1.65)$ & $1-6$ \\
\hline & Lack of control & $3.25(1.6)$ & $1-6$ \\
\hline & Reduction in other activities & $2.58(1.52)$ & $1-6$ \\
\hline & Time & $2.84(1.39)$ & $1-6$ \\
\hline & Intention & $2.95(1.66)$ & $1-6$ \\
\hline & Total (mean) & $3(1.14)$ & $1.10-5.43$ \\
\hline EDEQ compulsion & & $9.73(11.09)$ & $0-35$ \\
\hline \multirow{3}{*}{ Motivation } & Weight control & $3.85(1.33)$ & $1-6$ \\
\hline & Social reason & $2.55(1.02)$ & $1-4.67$ \\
\hline & Health reason & $4.26(1.36)$ & $1-6$ \\
\hline \multirow{6}{*}{ Physical self inventory } & Global self-concept & $3.5(0.68)$ & $1.6-5.2$ \\
\hline & Physical self-worth & $2.46(1.05)$ & $1-5.8$ \\
\hline & Physical condition & $3.07(0.72)$ & $1.75-4.8$ \\
\hline & Sport competences & $2.83(1.17)$ & $1-5.25$ \\
\hline & Attractive body & $3.27(0.79)$ & $1-4.33$ \\
\hline & Physical strength & $2.76(1.14)$ & $1-6$ \\
\hline
\end{tabular}


Table 1. (continued).

\begin{tabular}{llc}
\hline Test/survey & Item & Mean (SD) \\
\hline Sex & Female & $100(52)$ \\
& Min-max \\
& Never & $20.41(10)$ \\
Physical activity & Rarely & $32.65(16)$ \\
& Sometimes & $24.49(12)$ \\
& Often & $22.45(11)$ \\
& & $15.4(8)$ \\
Exercise dependence & Dependance & $63.4(32)$ \\
& Symptomatique & $19.23(10)$ \\
\hline
\end{tabular}

Table 2. Fit indices for the Latent Profile Analysis of the AM patients are presented in this table. From the left to the right: the entropy, loglikelihood (LL), Akaike, Bayesian, and adjusted Bayesian information criteria (AIE, BIC, aBIC, respectively), the LoMendell-Rubin adjusted Likelihood ratio test values (LMR $P$-value) and the Bootstrap Likelihood ratio test values (BLMT $P$-value) are given for each number of class model.

\begin{tabular}{lccccccc}
\hline Class-Model & Entropy & LL & AIC & BIC & aBIC & $\begin{array}{c}\text { LMR } \\
P \text {-value }\end{array}$ & $\begin{array}{c}\text { BLMT } \\
P \text {-value }\end{array}$ \\
\hline 1 Class & & & -672.33 & 1384.65 & 1419.40 & 1356.77 & $/$ \\
2 Class & 0.99 & -600.39 & 1262.77 & 1316.64 & 1219.57 & 0.016 \\
3 Class & 0.99 & -573.71 & 1231.42 & 1304.4 & 1172.66 & 0.38 & 0.0000 \\
4 Class & 0.97 & -573.71 & 1225.90 & 1318.00 & 1152 & 0.57 \\
\hline
\end{tabular}

and the quantity of physical activity measured with the GLTEQ (35.13 \pm 24.02$)$. High level of heterogeneity was also reported for the quality of life in the overall dimensions of the Duke health profile (with an average standard deviation equal to 25.26). To a lesser extent, such a result can also be reported in the PE measured with the EDS-R. Finally, the results coming from the EDS-R demonstrated that $15.4 \%$ of patients exhibited a proven dependence to physical activities, $63.46 \%$ of patients exhibited some dependence symptoms, and $19.23 \%$ of patients being asymptomatic.

Despite this heterogeneity within the considered population, the aim of this study was to categorize AN patients to allow programs of adapted physical activities as relevant as possible. To do that, a latent profile analysis was used on standard values.

\subsection{Three-class model have been selected for the latent profile analysis}

Four classes were estimated at first glance (please see Tab. 2). However, the model with 3 classes provided the best fit, as indicated by the lower AIC and aBIC. The LoMendell-Rubin adjusted LRT test has a $p$-value of 0.57 . These results at those tests suggested that three classes were sufficient and that four classes were not really needed. The bootstrapped parametric likelihood ratio test has a $p$-value $<0.0001$. So, it clearly meant that three classes were significantly better than two classes. Further- more, the entropy was still very good with three classes. However, the principle of parsimony could have pleaded in favour of two classes, because the indices were close to the five-class model. Nevertheless, by analysing the classes, the tree-class model made it possible to draw-up a very interesting global categorization profile: the patients who had a high exercise dependence scores, those who had low scores and those around the average. Finally, as it corresponded more to the clinical reality, the three-class model was chosen.

\subsection{Characteristics of the three profiles}

As shown in Table 3 and Figure 1, the three classes exhibited three different profiles with several differences between them. The first class included $21.4 \%$ of the patients, the second class included $21.4 \%$ and the third one $64.1 \%$ of the patients. The most important differences between them were content in $\mathrm{PE}$ with compulsive physical activity, perceived fitness, and mood, (more precisely, anxiety and depression measured by the Duke health profile).

\subsubsection{About the PE}

Interestingly, there was a significant difference between the 3 classes (please see Tab. 3). Post Hoc analysis exhibited that the first class presented compulsive physical activity scores significantly higher than the third 


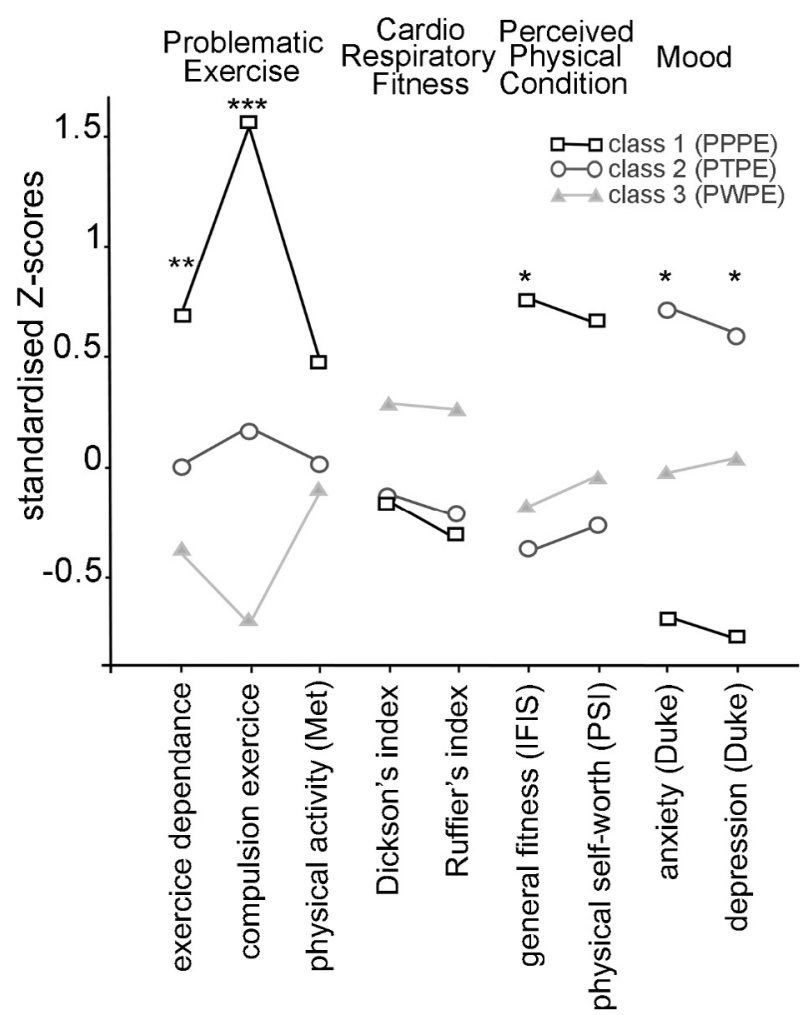

Fig. 1. Graphic of standard values of the Z-scores (y axis) for the most important items representing from left to right: the problematic exercises (exercise dependence, compulsion exercise, and physical activity frequency), cardio-respiratory fitness (Dickson's and Ruffier's index), perceived fitness (general fitness from the IFIS, physical self-worth from the PSI)) and mood (anxiety and depression from the Duke health profile). The first class exhibited by the latent profile analysis is plotted in black lines with square marks, the second class in dark grey with circle marks, and at last the third class is plotted in grey lines with triangle marks. Z score formula $=(x-\mu) / \sigma$ (with $x=$ individual's score; $\mu=$ general mean; $\sigma=$ general standard deviation). ${ }^{*} p$ value $<0.05 ;{ }^{* *} p$-value $<0.01 ;{ }^{* * *} p$-value $<0.001$.

class $[$ mean differences $=25.63 ; p<0.0001)]$, and the second class $[$ mean differences $=15.44 ; p<0.0001)]$. A significate difference was also present between class 2 and class 3 [mean differences $=10.19 ; p<0.0001$ )]. It was interesting to note that the first class presented the higher level of exercise dependence, significantly more important than the two others. Post Hoc analysis revealed that only the first class presented an exercise dependence score significantly higher than class 3 [mean differences $=1.27$; $p=0.0003)]$. Moreover, patients in the first class did significantly more physical activity than those in the two others, but only when physical activity was quantified in a frequency manner. Indeed, this significant difference was not exhibited by the GLTEQ score.

\subsubsection{About the psychological variables}

Significant differences between the first class and the two others concerned the score of perceived fitness, and mood (depression and anxiety) $([\mathrm{F}(2)=3.68, \quad p=0.03] ; \quad[\mathrm{F}$
$(2)=4.54, p=0.02] ; \quad[\mathrm{F}(2)=4.39, p=0.02]$, respectively). The first class exhibited a perceived fitness higher than the third and the second class [averaged difference (Class 1$3=0.85 ; p=0.05$ ); averaged difference (Class $1-2=1.06$; $p=0.08)$ ], while anxiety and depression remained significantly lower ( $p=0.01$ for anxiety and $p=0.02$ for depression).

To sum up, these results highlighted that patients in the first class presented a proven $\mathrm{PE}$ with higher perceived fitness and lower anxiety and depression compared to the others. Patients in the second class exhibited a moderate $\mathrm{PE}$, but the highest level of anxiety and depression. Finally, the patients in the third class did not showed any $\mathrm{PE}$, and medium scores in the other variables. This latent profile analysis made it thus possible to pass from a sample of patients with very heterogeneous behaviors as shown by the results of the different tests, to a categorization into 3 classes grouping each patient with common profiles. At this step, the first class (including $21.4 \%$ of the sample) characterized by a significant higher score at the Exercise Dependence Scale-Revised, at the compulsive practice, and a higher frequency of physical activity practice than the other two, will be named patients with proven $\mathrm{PE}$ (PPPE). The two other classes differed by their scores at the Exercise Dependence Scale-Revised and those at the compulsive exercise which were significantly higher in the second class. Consequently, the second patient profile (the second class, concerning $14.5 \%$ of the sample) will be named patients with a tendency to PE (PTPE). Finally, the third and last profile (the third class, concerning $64.1 \%$ of the sample) will be named patients without any PE (PWPE).

The aim was then now to identify specific guidelines for each category of patient that may enhance adapted physical activities treatment outcomes in AN patients. This was debated in the discussion section.

\section{Discussion}

The aim of this study was thus to highlight different profiles of patients in order to better adapt the contents of the physical activity programs proposed to AN patients. The pursuit of such an objective was recommended in the third principle of the reference work written by Cook et al. (2016). More precisely, these authors suggested to "screen for exercise-related psychopathology" in order to identify the patients who endorse pathological attitudes and behaviors towards physical exercise (i.e. exercise dependence, exercise addiction, and compulsive exercise). The latent profile analysis allowed to exhibit three classes corresponding to three different profiles reported to physical activity practices in female patients from 14 to 24 years old with a BMI from 13.05 to 20.76 . So the professional teacher in adapted physical activities (APA) will be able to propose APA programs (i) with common objectives for all the AN patients (for example: regain pleasure in physical activities, learn safe postures for a good physical activity practice, knowledge of their body...) but also, and that is a really novelty in the AN field, (ii) APA programs with very relevant contents specifically designed for each profile of patients. 
Table 3. Comparison of the three class resulting on the latent profile analysis: mean differences ( \pm standard deviation) and statistical analyses across the three classes are reported for problematic exercises (total of the exercise dependence, compulsion, and physical activity frequency), perceived fitness (general fitness from the IFIS, physical self-worth from the PSI) cardio-respiratory fitness (Dickson's index) and mood (anxiety and depression from the Duke health profile).

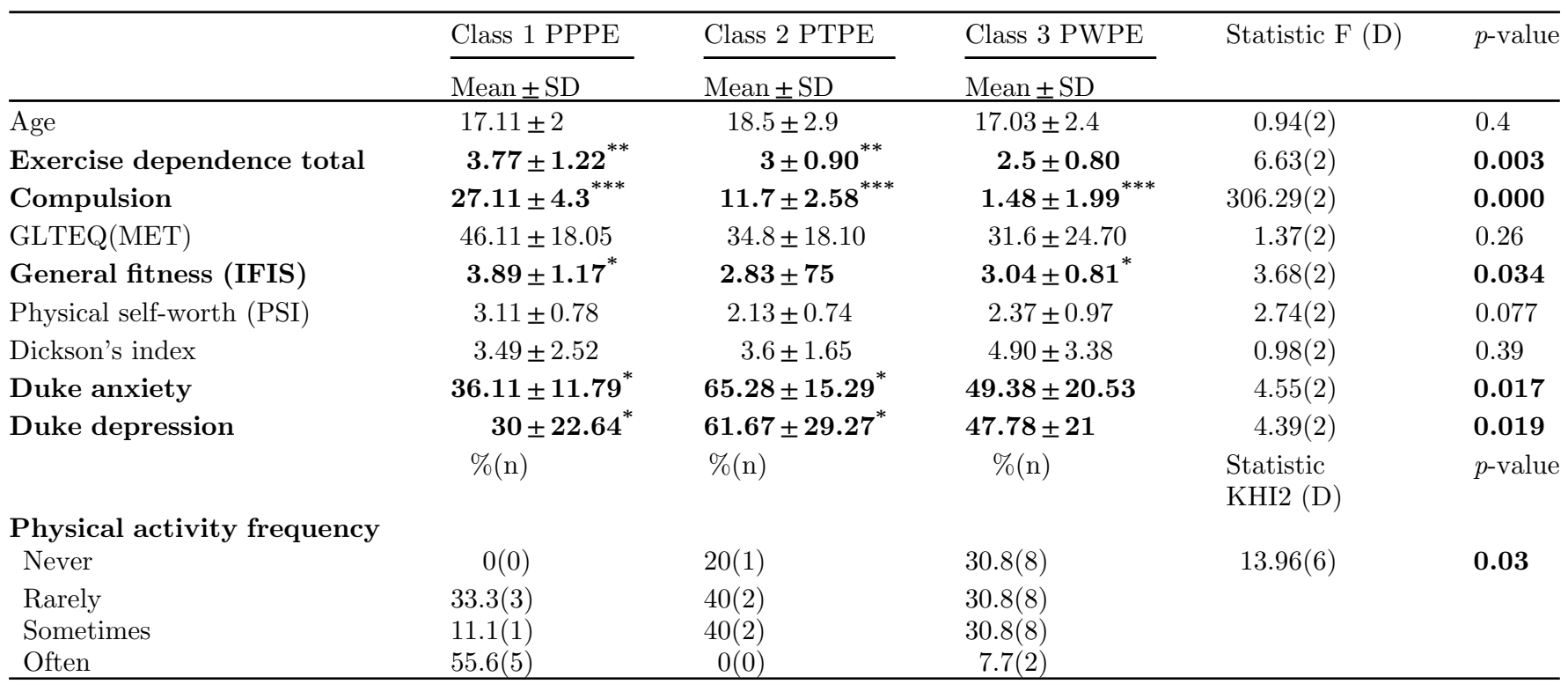

Parameters exhibiting a significant differences across at least two classes are reported in bold characters in a sake of clarity.

${ }^{*} p$-value $<0.05 ; * *$-value $<0.01 ; * *$ p-value $<0.001$.

In the present study, the percentage of AN patients suffering from PE was $21.4 \%$ of PPPE with an additional $14 \%$ of PTPE for a total ratio of $35.4 \%$ of the patient sample who had problematic relationships with physical activity. This ratio is in accordance with the literature in which from 5 to $54 \%$ of AN patients developed PE behaviors (Rizk et al., 2015). This wide range could be due to inconstant definitions for AN patients, inconstant definitions for PE, and several different tests and surveys to measure the PE. Considering solely the physical activity measured in Met by the GLTEQ, no significant difference was found in this study across the three classes. Significant differences appeared when physical activity was measured in frequency per week. But starting solely from this measure, it is impossible to know if there is $\mathrm{PE}$ or not. Measuring physical activity in AN patients remains really difficult. Every method presents some advantages but also limits, and no method has yet been published as perfect. In this study, the chosen and original method included all the parameters generally used to quantify and assay the PE and the mood of these patients, and analysed them in a combined way. That is the really new advantage of the present method. Every tools generally used in the literature to quantify the PE were included, completed with mood state, in order to draw a general assessment of patients from all these tests together.

About the PPPE category: It is important to note here that numerous studies had pointed PE as playing an important role in the etiology, development and perseverance of eating disorders (Renz et al., 2017; Rizk, Kern, \& Godart, 2014; Rizk et al., 2015). From the results of this study, in this first profile, the patients were characterized by their PE, but also by a depression and anxiety levels lower than the other patients. Physical activity is here a real addiction as defined by Goodman (1990), it provides relief from suffering and pleasure, and is constantly repeated independently of the consequences. The hypothesis is that physical activity in this group allows an effective emotional regulation but at the prize of frantic and compulsive practice. If this hypothesis is true, it then becomes easy to understand that constraining these patients to entirely stop physical activity would be probably lived in a violent way. Another hypothesis to explain these results would be that those with PE are those who are most in control, and therefore perhaps in denial. Moreover, despite a very high practice of physical activities, the Ruffier's and the Disckson's index, witnesses a general aerobic condition, were not significantly different from the other AN patients. In other words, this very high practice of physical activities did not allow a better cardiovascular fitness state, despite these patients perceived it as very satisfactory as revealed by the IFIS. As an all, patients in this PPPE category present proven and drastic $\mathrm{PE}$, a good perceived physical condition which is in fact not really better than that of other patients, and a quite good mood state according to the low depression and anxiety levels.Consequently, the main aim of the professional teacher in APA with PPPE should be an accompaniment of patients in their physical exercise in order to learn to manage this problematic relationship with physical practice. This major aim is characteristic and particularly relevant for this profile of patients. A focus should be on their real abilities and not on their perceived abilities. This would prevent these patients from 
engaging in risky physical activities, for example with postures causing potential injury when performed with an insufficient level of strength. In this vein, as recommended by Cook et al. (2016), the professional teacher in APA must put in place a real therapeutic education to help these patients better understand physical activity in order to preserve their physical integrity. Finally, the professional teacher in APA will propose physical situations stimulating some groups of muscles, or some metabolic chains set apart by the PPPE. So, it will reduce the risk of injury (Draeger, 2005) while regulating the mood affects, without the drastic negative consequences of putting the patient to a strict and forced state of rest. In addition to these principle physical objectives, some works on cognitive flexibility and self-esteem is often needed. Berczik, Szabo, Griffiths et al. (2012) also talk about learning self-control and moderation.

About the PTPE category: this second profile of AN patients corresponded to the second class revealed by the latent profile analysis in the result section. These are patients with a medium level of problematic relationship with physical activity, and with the highest depression and anxiety level. Relationships between physical activity, $\mathrm{PE}$, anxiety and depression have been studied in the literature. Some authors demonstrated that physical activities allowed AN patients to compensate for caloric intake as well as regulate mood (Noetel et al., 2016). This result is confirmed by the PPPE profile of patients in this paper. Other authors highlighted the very negative consequences of the interaction between a tendency to $\mathrm{PE}$ associated with depression and anxiety (Smith et al., 2013). Indeed, these authors clearly demonstrated that tendency to over-exercise and medium to high depression symptoms appeared to be significantly associated with suicidal behavior. According to these authors, tendency to $\mathrm{PE}$ and $\mathrm{PE}$ in AN patients allowed them to develop pain insensitivity which coupled with depression generate capability for suicide.

Consequently, the professional teacher in APA must put in place therapeutic education to first avoid aggravating the PE behavior. In this way, similar strategies to PPPE profile could be considered. Moreover for PTPE, a particular care should be taken to associate the physical practices with a pleasant, beneficial and controlled practice, and not as an outlet intended to burn caloric intake whatever the endured pain. Thereupon, the process of associating exteroceptive landmarks with interoceptive causes could enable PTPE to regain consciousness of their bodies. For example: "If I cannot talk anymore while I'm running, it's because the intensity of my run exceeds the first breath threshold and that the physical exercise becomes really intense, no matter how much pain I feel." About aerobic exercises, this example is all the more important as Bernstein \& McNally (2017) demonstrated that aerobic exercises at moderate intensity may help to attenuate negative emotions and are positive for mood. Moreover, this process would give patients knowledges in physical activity and would facilitate them to apply principles of self-control (Berczik et al., 2012). At last, several physical activities as pilates or yoga, generally unknown or neglected by the patients, are to be included for at least both reasons: first, it was proved that these physical activities reduce anxiety and depressive symptoms (Franklin, Butler, \& Bentley, 2018; McCall, 2018), which are one of the major pathological features of this PTPE patient profile. Second, these activities increase body satisfaction (Neumark-Sztainer, MacLehose, Watts, Pacanowski, \& Eisenberg, 2018), could improve strength in weak people or patients (Mazor et al., 2017), and solicit all the muscles (superficial and deep) in a global way (Rathore, Trivedi, Abraham, \& Sinha, 2017).

About the PWPE category: this third and last profile of AN patients corresponded to the third class revealed by the latent profile analysis in the result section. This PWPE do not present any problem with physical activity practice, and their Ruffier's or Dickson's index attest to a quite good ability to effort adaptation. The patients in this PWPE category presented an intermediate level of anxiety between the other 2 groups.

Consequently, the APA program, as a form of prevention, should aim to give the PWPE all the knowledge about the practice of moderate and safe physical activities, and advise about PE risks. The notions of pleasure and team work will be widely discussed, and the APA programs should also propose means of relaxation and well-being.

\section{Conclusion}

From heterogeneous sample of AN patients, the present study highlighted three categories of patients based from three typical pattern of psychopathological behaviors. Aiming to individualize and enhance physical activity programs, specific guidelines for each category of patients were then addressed. However, this study presented also some limits. First, despite the sample was correct from a statistical point of view (Muthén \& Muthén, 2002), it could be again more important for instance to extend analysis and result to wider age range or include men. Consequently, such a data base will continue to be extent. Secondly, no objective measure of physical activity was provided here. In this way, actimetric measures appear as a potentially promising tool. Once their reliability in AN patients more investigated, it will be necessary to include these kind of data in complement in such analyses. Thirdly, future studies will compared AN populations with control groups paired in gender and age. Finally, some adapted physical activity programs are being realized and their impacts will be studied.

A cknowledgements. The authors thank the Institut Mutualiste Montsouris, the Poissy pediatric hospital, the Nantes CHU, Renault de Tournemire (Nantes CHU), and all the patients who allowed us to realize the data acquisitions. We thank the Movement \& Sport Sciences review for this special issue dedicated to adapted physical activities and health. 


\section{References}

Almeida, M., Barra, A., Saltiel, F., Silva-Filho, A., Fonseca, A., \& Figueiredo, E. (2016). Urinary incontinence and other pelvic floor dysfunctions in female athletes in Brazil: A crosssectional study. Scandinavian Journal of Medicine $\&$ Science in Sports, 26(9), 1109-1116.

American Psychiatric Association. (2013). Diagnostic and statistical manual of mental disorders. Available from https://dsm.psychiatryonline.org/doi/abs/10.1176/appi. books.9780890420249.dsm-iv-tr.

Apor, P., Petrekanich, M., \& Számadó, J. (2009). Heart rate variability analysis in sports. Összefoglaló Referátumok, 150 (18), 847-853

Arcelus, J., Mitchell, A.J., Wales, J., \& Nielsen, S. (2011). Mortality rates in patients with anorexia nervosa and other eating disorders: a meta-analysis of 36 studies. Archives of General Psychiatry, 68(7), 724-731.

Berczik, K., Szabo, A., Griffiths, M.D., Kurimay, T., Kun, B., Urbán, R., Demetrovics, Z. (2012). Exercise addiction: symptoms, diagnosis, epidemiology, and etiology. Substance Use E Misuse, 47(4), 403-417.

Berlin, K.S., Williams, N.A., \& Parra, G.R. (2014). An introduction to latent variable mixture modeling (part 1): Overview and cross-sectional latent class and latent profile analyses. Journal of Pediatric Psychology, 39(2), 174-187.

Bernstein, E.E., \& McNally, R.J. (2017). Acute aerobic exercise helps overcome emotion regulation deficits. Cognition and Emotion, 31(4), 834-843.

Beumont, P.J., Arthur, B., Russell, J.D., \& Touyz, S.W. (1994). Excessive physical activity in dieting disorder patients: Proposals for a supervised exercise program. International Journal of Eating Disorders, 15, 21-36.

Boutin, R.D., White, L.M., Laor, T., Spitz, D.J., Lopez-Ben, R. R., Stevens, K.J., \& Bredella, M.A. (2015). MRI findings of serous atrophy of bone marrow and associated complications. European Radiology, 25(9), 2771-2778.

Calogero, R., \& Pedrotty, K. (2004). The practice and process of healthy exercise: An investigation of the treatment of exercise abuse in women with eating disorders. Eating Disorders, 112, 273-291.

Chantler, I., Szabo, C., \& Green, K. (2006). Muscular strength changes in hospitalized anorexic patients after an eight week resistance training program. International Journal of Sports Medecine, 27(08), 660-665.

Cook, B.J., Wonderlich, S.A., Mitchell, J.E., Thompson, R., Sherman, R., \& Mccallum, K. (2016). Exercise in eating disorders treatment: systematic review and proposal of guidelines. Medicine $\&$ Science in Sports $\&$ Exercise, 48(7), 1408-1414.

Dalle Grave, R., Calugi, S., \& Marchesini, G. (2008). Compulsive exercise to control shape or weight in eating disorders: Prevalence, associated features, and treatment outcome. Comprehensive Psychiatry, 49(4), 346-352.

Davis, C., Katzman, D.K., Kaptein, S., Kirsh, C., Brewer, H. Kalmbach, K., Olmsted, M.P., Woodside, D.B., \& Kaplan, A.S. (1997). The prevalence of high-level exercise in the eating disorders: etiological implications. Comprehensive Psychiatry, 38(6), 321-326.

Drabkin, A., Rothman, M.S., Wassenaar, E., Mascolo, M., \& Mehler, P.S. (2017). Assessment and clinical management of bone disease in adults with eating disorders: a review. Journal of Eating Disorders, 5(1), 42.

Draeger, J. (2005). The obligatory exerciser. The Physician and Sportsmedicine, 33(6), 13-23.
Ehrlich, S., Burghardt, R., Weiss, D., Salbach-Andrae, H., Craciun, E.M., Goldhahn, K., Klapp, B.F., \& Lehmkuhl, U. (2008). Glial and neuronal damage markers in patients with anorexia nervosa. Journal of Neural Transmission, 115(6), 921-927.

El Ghoch, M., Calugi, S., Pellegrini, M., Milanese, C., Busacchi, M., Battistini, N.C., Bernabé, J., \& Dalle Grave, R. (2013). Measured physical activity in anorexia nervosa: features and treatment outcome. International Journal of Eating Disorders, 46(7), 709-712.

El Ghoch, M., Bazzani, P., \& Dalle Grave, R. (2014). Management of ischiopubic stress fracture in patients with anorexia nervosa and excessive compulsive exercising. BMJ Case Reports, 2014, 1-4.

El Ghoch, M., Calugi, S., Pellegrini, M., Chignola, E., \& Dalle Grave, R. (2016). Physical activity, body weight, and resumption of menses in anorexia nervosa. Psychiatry Research, 246, 507-511.

Faik, A., Vanderhulst, E., Rossem, V.I., \& Devroey, D. (2017). Influence of physical activity and interest for food and sciences versus weight disorders in children aged 8 to 18 years. Journal of Preventive Medicine and Hygiene, 58(2), E105.

Fairburn, C., \& Beglin, S. (1994). Assessment of eating disorders: Interview or self-report questionnaire? International Journal of Eating Disorders, 16, 363-370.

Fernandez-del-Valle, M., Larumbe-Zabala, E., Villaseñor-Montarroso, A., Cardona Gonzalez, C., Diez-Vega, I., Lopez Mojares, L.M., \& Perez Ruiz, M. (2014). Resistance training enhances muscular performance in patients with anorexia nervosa: a randomized controlled trial. International Journal of Eating Disorders, 47(6), 601-609.

Fox, K.R., \& Corbin, C.B. (1989). The physical self-perception profile: development and preliminary validation. Journal of Sport \& Exercise Psychology, 11(4), 408-430.

Franklin, R.A., Butler, M.P., \& Bentley, J.A. (2018). The physical postures of yoga practices may protect against depressive symptoms, even as life stressors increase: a moderation analysis. Psychology, Health 83 Medicine, 23(7), 870-879.

Franko, D.L., Keshaviah, A., Eddy, K.T., Krishna, M., Davis, M. C., Keel, P.K., \& Herzog, D.B. (2013). A longitudinal investigation of mortality in anorexia nervosa and bulimia nervosa. American Journal of Psychiatry, 170(8), 917-925.

Godin, G. (2011). Commentary : The godin-shephard leisuretime physical activity questionnaire. Health $\&$ Fitness Journal of Canada, 4(1), 5.

Godin, G., Jobin, J., \& Bouillon, J. (1986). Assessment of leisure time exercise behavior by self-report: a concurrent validity study. Canadian Journal of Public Health, 7ry(5), 359-362.

Goodman, A. (1990). Addiction: definition and implications. British Journal of Addiction, 85(11), 1403-1408.

Gottschall, J.S., Mills, J., \& Hastings, B. (2013). Integration core exercises elicit greater muscle activation than isolation exercises. The Journal of Strength $\&$ Conditioning Research, 27(3), 590-596.

Gouju, J.-L. (1993). Course d'endurance, didactique et motivation. Revue eps, 241, 54-57.

Guillemin, F., Paul-Dauphin, A., Virion, J.-M., Bouchet, C., \& Briançon, S. (1997). Le profil de santé de DUKE : un instrument générique de mesure de qualité de vie liée à la santé. Santé Publique, 1, 35-44.

Hausenblas, H.A., \& Downs, D.S. (2002). Exercise dependence: A systematic review. Psychology of Sport and Exercise, 3, $89-123$

Hausenblas, H.A., Cook, B.J., \& Chittester, N.I. (2008). Can exercise treat eating disorders? Exercise and Sport Sciences Review, 36(1), 43-47. 
Hebebrand, J., Exner, C., Hebebrand, K., Holtkamp, C., Casper, R., Remschmidt, H., Herpetz-Dahlmann, B., \& Klingenspor, M. (2003). Hyperactivity in patients with anorexia nervosa and in semistarved rats: evidence for a pivotal role of hypoleptinemia. Physiology $\&$ Behavior, 79(1), 25-37.

Joussellin, E. (2007). Filetest de Ruffier, improprement appelé test de Ruffier-Dickson. Médicins du sport, 83(4), 33-34.

Junne, F., Zipfel, S., Wild, B., Martus, P., Giel, K., Resmark, G., Friederich, H-C., Teufel, M., De Zwaan, M., Dinkel, A., Herpertz, S., Burgmer, M., Tagay, S., Rothermund, E., Zeeck, A., Ziser, K., Herzog, W., \& Löwe, B. (2016). The relationship of body image with symptoms of depression and anxiety in patients with anorexia nervosa during outpatient psychotherapy: Results of the ANTOP study. Psychotherapy, $53(2), 141$.

Kern, L. (2007). Dépendance et exercice physique : une échelle de dépendance à l'exercice physique. Psychologie Française, 52, 403-416.

Kolnes, L.-J. (2017). Exercise and physical therapy help restore body and self in clients with severe anorexia nervosa. Journal of Bodywork and Movement Therapies, 21(3), 481-494.

Krantz, M.J., Gaudiani, J.L., Johnson, V.W., \& Mehler, P.S. (2011). Exercise electrocardiography extinguishes persistent junctional rhythm in a patient with severe anorexia nervosa. Cardiology, 120(4), 217-220.

Kuwabara, M., Niwa, K., Yamada, U., \& Ohta, D. (2017). Low body mass index correlates with low left ventricular mass index in patients with severe anorexia nervosa. Heart and Vessels, 33(1), 89-93.

Lands, L., Pavilanis, A., Charge, T.D., \& Coates, A.L. (1992). Cardiopulmonary response to exercise in anorexia nervosa. Pediatric Pulmonology, 13(2), 101-107.

Lawler, M., Heary, C., \& Nixon, E. (2017). Variations in adolescents' motivational characteristics across gender and physical activity patterns: A latent class analysis approach. BMC Public Health, 17(1), 661-674.

Leblé, N., Radon, L., Rabot, M., \& Godart, N. (2017). Depressive symptoms during anorexia nervosa: State of the art and consequences for an appropriate use of antidepressants. Encephale, 43(1), 62-68.

Lisman, P., O'Connor, F.G., Deuster, P.A., \& Knapik, J.J. (2013). Functional movement screen and aerobic fitness predict injuries in military training. Medicine $\&$ Science in Sports 8 Exercise, 45(4), 636-643.

Mangerud, W.L., Bjerkeset, O., Lydersen, S., \& Indredavik, M.S. (2014). Physical activity in adolescents with psychiatric disorders and in the general population. Child and Adolescent Psychiatry and Mental Health, 8(1), 2.

Mazor, M., Lee, J.Q., Peled, A., Zerzan, S., Irwin, C., Chesney, M.A., Serrurier, K., Sbitany, H., Dhruva, A., Sacks, D., \& Smoot, B. (2017). The effect of yoga on arm volume, strength, and range of motion in women at risk for breast cancer-related lymphedema. The Journal of Alternative and Complementary Medicine, 154-160, 1-7.

McCall, M. (2018). Yoga intervention may improve healthrelated quality of life (HRQL), fatigue, depression, anxiety and sleep in patients with breast cancer. Evidence-based nursing, 21(1), 9-9.

McCreary, E.K., \& Provance, P.G. (1993). Muscles, testing and function: with, posture and pain. Baltimore: Williams \& Wilkins.

Mueller, S.M., Immoos, M., Anliker, E., Drobnjak, S., Boutellier, U., \& Toigo, M. (2015). Reduced bone strength and muscle force in women 27 years after anorexia nervosa. The Journal of Clinical Endocrinology \& Metabolism,100(8), 2927-2933.
Muthén, L.K., \& Muthén, B.O. (2002). How to use a Monte Carlo study to decide on sample size and determine power. Structural Equation Modeling, 9(4), 599-620.

Muthén L.K., Muthén B.O. (2015). Mplus User's Guide. Muthén \& Muthén, Los Angeles, CA: 1998-2012.

Neumark-Sztainer, D., MacLehose, R.F., Watts, A.W., Pacanowski, C.R., \& Eisenberg, M.E. (2018). Yoga and body image: Findings from a large population-based study of young adults. Body Image, 24, 69-75.

Ng, L., Ng, D., \& Wong, W. (2013). Is supervised exercise training safe in patients with anorexia nervosa? A metaanalysis. Physiotherapy, 99(1), 1-11.

Ninot, G., Delignières, D., \& Fortes, M. (2000). L'évaluation de l'estime de soi dans le domaine corporel. Staps, 53, 35-48.

Noetel, M., Miskovic-Wheatley, J., Crosby, R.D., Hay, P., Madden, S., \& Touyz, S. (2016). A clinical profile of compulsive exercise in adolescent inpatients with anorexia nervosa. Journal of Eating Disorders, 4(1), 1.

Nolte, K., Krüger, P.E., Els, P.S., \& Nolte, H. (2013). Three dimensional musculoskeletal modelling of the abdominal crunch resistance training exercise. Journal of Sports Sciences, 31(3), 264-275.

Ortega, F.B., Ruiz, J.R., España-Romero, V., Vicente-Rodriguez, G., Martínez-Gómez, D., Manios, Y., Béghin, L., Molanr, D., Widhalm, K., Moreno, L., \& Sjöström, M. (2011). The international fitness scale (IFIS): usefulness of self-reported fitness in youth. International Journal of Epidemiology, 40(3), 701-711.

Parkerson, G.J., Broadhead, W., \& Tse, C. (1990). The Duke health profile. A 17-item measure of health and dysfunction. Medical Care, 28(11), 1056-1072.

Patton, G., Coffey, C., \& Sawyer, S. (2003). The outcome of adolescent eating disorders: findings from the Victorian adolescent health cohort study. European Child \& Adolescent Psychiatry, 12(1), i25-i29.

Rathore, M., Trivedi, S., Abraham, J., \& Sinha, M.B. (2017). Anatomical correlation of core muscle activation in different yogic postures. International Journal of Yoga, 10(2), 59.

Renz, J.A., Fisher, M., Vidair, H.B., Hirsch, D., Malizio, J., Barger, H., \& Fornari, V. (2017). Excessive exercise among adolescents with eating disorders: examination of psychological and demographic variables. International Journal of Adolescent Medicine and Health, Available from pii:/j/ijamh. ahead-of-print/ijamh-2017-0032/ijamh-2017-0032.xml.

Rizk, M. (2015). Implication de l'hyperactivité physique dans l'anorexie mentale. Paris: Paris Descartes University.

Rizk, M., Kern, L., \& Godart, N. (2014). Physical activity and health: The positive and negative effects of physical exercise. Paper presented at the Fifth International Congress of Sport Psychology-SFPS, Nice, France.

Rizk, M., Kern, L., Godart, N., \& Melchior, J.-C. (2014). Anorexie mentale, activité physique et nutrition: quelles potentialisations? Nutrition Clinique et Métabolisme, 28(4), 287-293.

Rizk, M., Lalanne, C., Berthoz, S., Kern, L., Godart, N., \& Group, E. (2015). Problematic exercise in anorexia nervosa: Testing potential risk factors against different definitions. PLoS ONE, 10(11), 1-18.

Smith, A.R., Fink, E.L., Anestis, M.D., Ribeiro, J.D., Gordon, K. H., Davis, H., Keel, P.K., Bardone-Cone, A.M., Peterson, C. B., Klein, M.H., Crow, S., Mitchell, J.E., Crosby, R.D., Wonderlich, S.A., le Grange, D., \& Joiner, T.E. Jr. (2013). Exercise caution: over-exercise is associated with suicidality among individuals with disordered eating. Psychiatry Research, 206(2), 246-255. 
Steinhausen, H.-C. (2009). Outcome of eating disorders. Child and Adolescent Psychiatric Clinics of North America, 18(1), $225-242$.

Stheneur, C., Bergeron, S.J., Frappier, J.-Y., Jamoulle, O., Taddeo, D., Sznajder, M., \& Lapeyraque, A.-L. (2017). Renal injury in pediatric anorexia nervosa: a retrospective study. Eating and Weight Disorders-Studies on Anorexia, Bulimia and Obesity, doi: 10.1007/s40519-017-0401-1.

Sung, V.W., \& Hampton, B.S. (2009). Epidemiology of pelvic floor dysfunction. Obstetrics and Gynecology Clinics of North America, 36(3), 421-443.

Urhausen, A., Gabriel, H., Weiler, B., \& Kindermann, W. (1998). Ergometric and psychological findings during overtraining: a long-term follow-up study in endurance athletes. International Journal of Sports Medecine, 19(02), 114-120.

van Elburg, A., \& Danner, U. (2015). Anorexia nervosa en adolescenten. Tijdschrift voor psychiatrie, 57(12), 923-927.

Vancampfort, D., Vanderlinden, J., De Hert, M., Soundy, A., Adamkova, M., Skjaerven, L.H., Catalan-Matamoros, D.,
Lundvik Gyllensten, A., Gomez-Conesa, A., \& Probst, M. (2014). A systematic review of physical therapy interventions for patients with anorexia and bulemia nervosa. Disability and Rehabilitation, 36(8), 628-634.

Vitton, V., Baumstarck-Barrau, K., Brardjanian, S., Caballe, I., Bouvier, M., \& Grimaud, J.-C. (2011). Impact of highlevel sport practice on anal incontinence in a healthy young female population. Journal of Women's Health, 20(5), $757-763$.

Woodward, K., McIlwain, D., \& Mond, J. (2017). Feelings about the self and body in eating disturbances: The role of internalized shame, self-esteem, externalized self-perceptions, and body shame. Self and Identity, doi: 10.1080/ 15298868.2017.1403373.

Young, S., Rhodes, P., Touyz, S., \& Hay, P. (2013). The relationship between obsessive-compulsive personality disorder traits, obsessive-compulsive disorder and excessive exercise in patients with anorexia nervosa: a systematic review. Journal of Eating Disorders, 1(1), 16.

Cite this article as: Kern L, Godart N, Tailhardat L, Peguet A, Grall-Bronnec M, \& Fautrelle L (2018) A latent profile analysis revealed different categories of patients in anorexia nervosa: proposal of guidelines in adapted physical activity. Mov Sport Sci/Sci Mot, https://doi.org/10.1051/sm/2018022 